\section{Structure of Line Spectra in Crystals}

New investigations of the spectra of chromium in crystals ${ }^{1}$ have rendered very probable the surmise expressed by $\mathrm{Saha}^{2}$ as to how these spectra originate. The possibility is thus afforded of enlarg. ing the explanation proposed previously ${ }^{3}$ resulting in the main characteristics for a transition of an ing spectra of absorption determined by J. Becquerel, as I gather from a discussion with Mr. Gorter, of Harlem. The temperature behaviour of the lines is as expected (strong dependence on temperature of ${ }^{3} F-{ }^{8} H$ in relation to ${ }^{1} G-{ }^{3} H$ in praseodymium). Also the appearance of the transitions leading to the deepest level in absorption is fulfilled. As regards the intensities of the single lines, the transitions

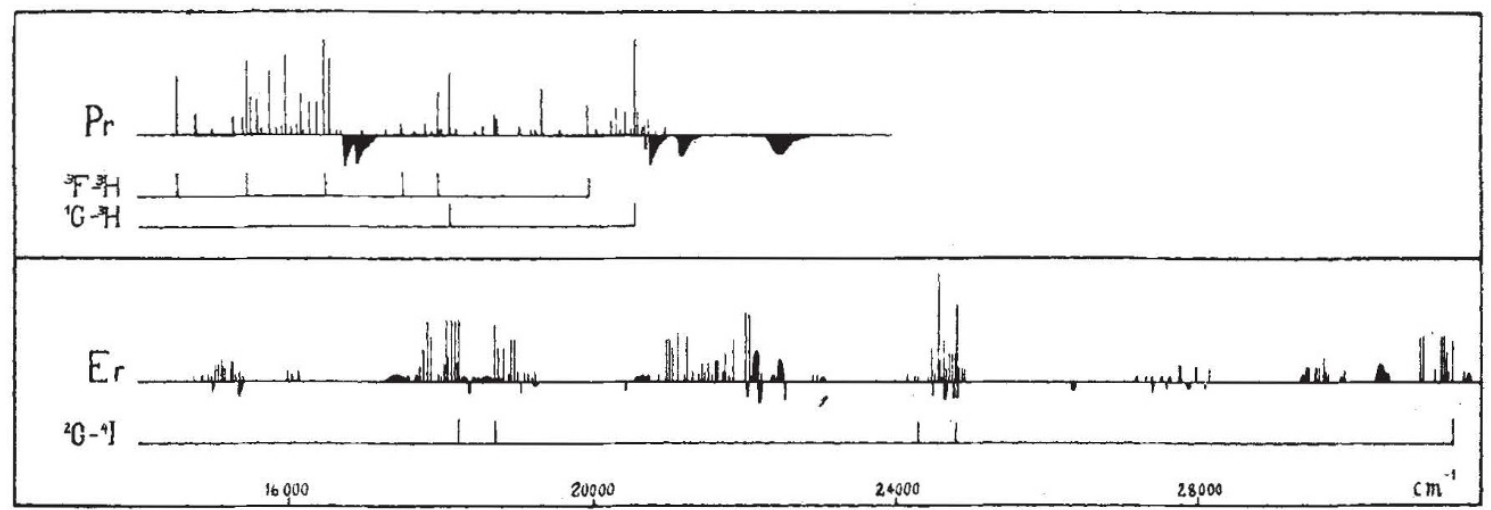

FIa. 1.

electron and its change through the crystal lattice, inasmuch as now also the laws of the various transitions of electrons for the spectra of phosphorescence of the rare earths can be investigated. The explanation of transitions $4 f^{z \leftrightarrow 4 f^{z-1}} 5 d$ offered by Laporte ${ }^{4}$ should come into consideration only for the diffuse spectra of the rare earths, for example, of neodymium (also of samarium ${ }^{5}$ ).

In analogy with chromium we might attribute the sharp spectra to transitions without change of the electronic configurations. Then the principal transitions of electrons appearing in the respective spectra must be attributed to the multiplets produced by the combination of the possible terms of the unchanged electronic configurations. The separa. tion of these terms has been calculated according to a method indicated by Goudsmit ${ }^{6}$, and the single term intervals have been fixed according to the Landé interval rule. A very good concordance of the values calculated with the strongest lines of the emission spectrum, for example, in $\mathrm{CaO}$, becomes manifest, as shown by Fig. I with the example of praseodymium and erbium, the values of which are indicated according to my own investigations and those of Evert and Fagerberg. The remaining lines originate in accordance with the former explanation partly from lattice oscillations and perhaps also molecular oscillations, partly also from Stark effects.

As these lengthy calculations of the splitting of the terms have not yet been carried out for all electron arrangements, the following combinations have been found up to now :

Pr: ${ }^{3} F-{ }^{3} H,{ }^{1} G-{ }^{3} H,\left({ }^{1} S-{ }^{3} P\right)$

$\mathrm{Nd}:{ }^{2} I-4 I,{ }^{2} G-{ }^{4} I$

$\mathrm{Sm}:{ }^{\theta} F-{ }^{\theta} H$

Er : ${ }^{2} G-{ }^{4} I,\left({ }^{2} K-{ }^{4} I\right)$

The transitions without change of the spin are situated at longer waves than the corresponding ones with change of the spin. The screening constants are $\sigma_{2}=33.0 \pm 0.2$ for praseodymium, neodymium, samarium and erbium without appreciable direction of the different values.

The possibility of such transitions corresponds very well to the oscillator strength of the correspond.
$\Delta J=\Delta L$ are, so far as it is possible to judge qualitatively, mostly the strongest, even when $\Delta J=\Delta L= \pm 2$. R. TOMaschro.

Physical Institute of the University, Marburg-on-Lahn. Sept. 26.

1 O. Deutschbein, Z. Phys., 7\%, 492; 1932.

2 NATURE, 125, 163, Feb. 1, 1930.

R. Tomaschek, Z. Elektrochemie, 38, 737; 1930.

- Phys. Rev., 35, 30 1930.

R. Tomaschek, Phys. Z., 1932.

- Phys. Rev., 31, 946; 1928.

Anomalous Behaviour of Methane in the Raman Effect

DrCkinson, Dillon and Rasetti, ${ }^{1}$ who examined the Raman spectrum of methane, did not report the presence in it of any pure rotational lines accompanying the Rayleigh scattering. To decide whether indeed the rotational scattering is absent in the case of this gas, spectrograms were obtained by giving prolonged exposures up to a week, but without recording even a trace of such seattering by way of either discrete lines or unresolved bands alongside the undisplaced lines of the mercury spectrum. Special devices for eliminating stray light and weakening the Rayleigh scattering with the aid of a nicol were employed in order to secure the most favourable conditions for the observation, but these made no difference to the result stated.

The absence of a rotational scattering, which is thus indicated in the case of methane, is an extremely surprising result, as it is well known that the Rayleigh scattering by the molecules of this gas is depolarised to an appreciable extent ${ }^{2}(1 \cdot 14$ per cent), and theory indicates a direct connexion between the magnitude of such depolarisation and the intensity of the rotational Raman lines. Moreover, gases such as hydrogen chloride, ammonia, and carbon monoxide which show comparable or even smaller depolarisation values have been observed to exhibit strong rotational scattering.

210 Bow Bazar Street, S. BHAGavantaM. Oct. 8 .

Phys. Rev., 84, $582 ; 1929$.

No. 3289 , Vor. 130] 